\title{
Differential and maximal ideals of the ultrametric Corona algebra
}

\author{
Alain Escassut \\ Dedicated to the memory of Nicole De Grande-De Kimpe
}

\begin{abstract}
Let $K$ be a complete ultrametric algebraically closed field and let $A$ be the Banach $K$-algebra of bounded analytic functions in the "open" unit disk $D$ of $K$ provided with the Gauss norm. Maximal ideals of infinite codimension are examined in connection with ultrafilters on $D$. Four classes of ultrafilters on $D$ are considered, defining a null ideal, or a maximal ideal or an unidentified ideal.

A function $f \in A$ tends to 0 along a sequence of disks $\left|x-a_{n}\right|<r$ with $\lim _{n \rightarrow \infty}\left|a_{n}\right|=1$ if and only if so does $f^{(k)}$ for every $k \in \mathbb{N}$. If $K$ is spherically complete, the ideal defined by an ultrafilter either is a maximal ideal or is differential. In many cases, it is not both maximal and differential. If two ultrafilters are not contiguous, and if they define non-differential ideals, then they define two distinct maximal ideals. Unsolved problems are presented.
\end{abstract}

\section{Introduction and results}

Let $K$ be an algebraically closed field complete with respect to an ultrametric absolute value |. . Given $a \in K$ and $r>0$, we denote by $d(a, r)$ the disk $\{x \in K|| x-a \mid \leq r\}$, by $d\left(a, r^{-}\right)$the disk $\{x \in K|| x-a \mid<r\}$ and set $D=d\left(0,1^{-}\right)$. Let $A$ be the $K$-algebra of bounded power series converging in $D$ which is complete with respect to the Gauss norm defined as $\left\|\sum_{n=1}^{\infty} a_{n} x^{n}\right\|=\sup _{n \in \mathbb{N}}\left|a_{n}\right|$. We know that this norm of $A$ actually is the norm of uniform convergence on $D$ [5].

The Corona Problem in complex analysis [4] consisted of proving that the set of maximal ideals defined by points of the open unit disk is dense in the whole maximal spectrum with respect to the Gelfand topology. In our algebra $A$, the maximal ideals that are not defined by points of $D$ actually are of infinite codimension. Consequently, there is no relevant topology on the maximal spectrum. However, the set of continuous multiplicative semi-norms, also called multiplicative spectrum, plays a similar role, provided with the topology of pointwise convergence [12], [2], $[6]$.

1991 Mathematics Subject Classification. Primary 12J25 Secondary 46S10. ideals.

Key words and phrases. p-adic analytic functions, ultrametric corona problem, maximal 
In [7], [9], [10] we characterized all continuous multiplicative norms of $A$ and we saw that the mystery only comes from multiplicative semi-norms whose restriction to polynomials is the Gauss norm but have a non-zero kernel consisting of functions admitting infinitely many zeroes. Here this paper is aimed at examining maximal ideals of infinite codimension of $A$ and their links with ultrafilters on $D$.

Definition: An ideal $\mathcal{I}$ of $A$ is said to be differential if for every $f \in A, f^{(k)}$ belongs to $A$ for all $k \in \mathbb{N}$.

In [14], the question whether there exist differential maximal ideals was asked. Here we will examine connections between maximal ideals and differential ideals.

Describing continuous multiplicative semi-norms requires to recall several definitions concerning circular filters.

Let $a \in K$ and $r^{\prime}$, $r^{\prime \prime}$ with $0<r^{\prime}<r^{\prime \prime}$ We denote by $\Gamma\left(a, r^{\prime}, r^{\prime \prime}\right)$ the annulus $\left\{x \in K\left|r^{\prime}<\right| x-a \mid<r^{\prime \prime}\right\}$. Now, let $r$ be $>0$. We call circular filter of center $a$ and diameter $r$ the filter admitting as a generating system the set of annuli $\Gamma\left(b, r^{\prime}, r^{\prime \prime}\right)$ with $b \in d(a, r)$ and $r^{\prime}<r<r^{\prime \prime}$.

Recall that the field $K$ is said to be spherically complete if every decreasing sequence of disks has a non-empty intersection.

In a field which is not spherically complete, one has to consider decreasing sequences of disks $\left(D_{n}\right)$ with an empty intersection. We call circular filter with no center, of canonical basis $\left(D_{n}\right)$ the filter admitting for basis the sequence $\left(D_{n}\right)$ and the number $\lim _{n \rightarrow \infty} \operatorname{diam}\left(D_{n}\right)$ is called diameter of the filter

Finally the filter of neighborhoods of a point $a \in D$ is called circular filter of the neighborhoods of a on $D$ and its diameter is 0 .

Circular filters on $K$ are well known to characterize multiplicative semi-norms on $K[x]$ [5], [6], [12]. Particularly, circular filters of strictly positive diameter are known to characterize absolute values on $K[x]$.

By continuity, each circular filter of strictly positive diameter $\mathcal{F}$ that is secant with $D$ also defines a continuous absolute value $\varphi_{\mathcal{F}}(f)=\lim _{\mathcal{F}}|f(x)|$ and each point $a \in D$, of course defines a continuous multiplicative semi-norm $\varphi_{a}$ as $\varphi_{a}(f)=|f(a)|$. Let $\operatorname{Mult}(A, \|$. \|) be the set of all continuous multiplicative semi-norms of $A$, let $\operatorname{Mult}_{m}(A, \|$. $\|)$ be the set of continuous multiplicative semi-norms of $A$ whose kernel is a maximal ideal and let $\operatorname{Mult}_{a}(A,\|\|$.$) the set of continuous multiplicative$ semi-norms of $A$ of the form $\varphi_{a}$. The set $\operatorname{Mult}(A,\|\cdot\|)$ is provided with the topology of simple convergence.

Here, we shall denote by $\mathcal{W}$ the circular filter of center 0 and diameter 1 . A filter on $D$ will be said to be coroner if it is thinner than $\mathcal{W}$ and a sequence $\left(a_{n}\right)$ of $D$ will be said to be coroner if $\lim _{n \rightarrow \infty}\left|a_{n}\right|=1$. In the same way, a sequence of disks $\left(d\left(a_{n}, r\right)_{n \in \mathbb{N}}\right)$ will be said to be coroner if the sequence $\left(a_{n}\right)_{n \in \mathbb{N}}$ is coroner.

Every ultrafilter $\mathcal{U}$ on $D$ defines an element $\varphi_{\mathcal{U}}$ of $\operatorname{Mult}(A,\|\|$.$) as \varphi_{\mathcal{U}}(f)=$ $\lim _{\mathcal{U}}|f(x)|$ : such a limit does exist because the functions $f \in A$ are bounded and therefore $|f(x)|$ takes values in the compact $[0,\|f\|]$. And such an element obviously belongs to the closure of $\operatorname{Mult}_{a}(A, \|$. \|) in $\operatorname{Mult}(A, \|$. $\|)$. However, the only ultrafilters on $D$ we have to consider are the coroner ultrafilters.

Given a filter $\mathcal{F}$ on $D$ we will denote by $\mathcal{J}(\mathcal{F})$ the ideal of the $f \in A$ such that $\lim _{\mathcal{F}} f(x)=0$. 
In [7] it is proved that each continuous absolute value on $A$ whose restriction to $K[x]$ is of the form $\varphi_{\mathcal{F}}$ with $\mathcal{F}$ a circular filter on $D$ of diameter $r<1$, has a unique continuation to $A$ and, as said above, this continuation is of the form $\varphi_{\mathcal{F}}(f)=\lim _{\mathcal{F}}|f(x)|$. On the other hand, the continuous absolute value $\varphi_{\mathcal{W}}$, as an absolute value on $K[x]$, admits infinitely many continuations on $A$, not only as the Gauss norm, but also in the form $\varphi_{\mathcal{U}}(f)=\lim _{\mathcal{U}}|f(x)|$ whereas $\mathcal{U}$ is an ultrafilter thinner than $\mathcal{W}$ while $\mathcal{J}(\mathcal{U})$ is a maximal ideal of $A$. But in [9] it is proven that the only continuous absolute value on $A$ whose restriction to polynomials is the Gauss norm on $K[x]$, actually is the Gauss norm on $A$ again. And then, the Gauss norm is defined on the whole ajgebra $A$ in the following way. Consider the family of sequences of disks $\left(d\left(a_{n},\left|a_{n}\right|^{-}\right)_{n \in \mathbb{N}}\right)$ such that $\lim _{n \rightarrow+\infty}\left|a_{n}\right|=1$. This is a generating system of a filter thinner than $\mathcal{W}$ but for each function $f \in A,|f(x)|$ admits a limit along $\mathcal{Y}$ and that limit is the Gauss norm of $f$ [7] (Corollary 2.1).

Thus, we can ask whether there exist multiplicative semi-norms whose kernel is a prime closed ideal different from (0) that is not a maximal ideal. In [9] we showed the existence of prime closed ideals that are neither null nor maximal ideals. However, such ideals do not seem to be the kernel of continuous multiplicative seminorms. Here, thanks to J. Araujo's work, it appears that there do exist continuous multiplicative semi-norms whose kernel is a prime closed ideal that is neither null nor a maximal ideal [1].

On the other hand, the "ultrametric Corona problem" may be viewed at two levels:

$1)$ is $\operatorname{Mult}_{a}(A,\|\|$.$) dense in \operatorname{Mult}_{m}(A,\|\|$.$) ?$

$2)$ is it dense in $\operatorname{Mult}(A,\|\|$.$) ?$

In [9] we partially answered the first question by showing that if $K$ is spherically complete, then $\operatorname{Mult}_{a}(A, \|$. $\|)$ is dense in $\operatorname{Mult}_{m}(A,\|\|$.$) .$

Here we mean to thoroughly study maximal ideals of $A$ that are not of codimension 1. It is well known that maximal ideals of codimension 1 are of the form $(x-a) A$ with $a \in D[8]$. In [7] it is shown that a maximal ideal of infinite codimension of $A$ is of the form $\mathcal{J}(\mathcal{U})$ with $\mathcal{U}$ a coroner ultrafilter. But many coroner ultrafilters $\mathcal{U}$ only define an ideal $\mathcal{J}(\mathcal{U})$ that is null. An interesting question is to characterize the coroner ultrafilters $\mathcal{U}$ such that $\mathcal{J}(\mathcal{U})$ is a maximal ideal, or the null ideal (or any else prime ideal).

We will denote by $\mathcal{Y}$ the coroner filter generated by sets of the form $\Gamma(0, r, 1) \backslash$ $\left(\bigcup_{n=1}^{\infty} d\left(a_{n},\left|a_{n}\right|^{-}\right)\right)$where the sequences $\left(a_{n}\right)$ are coroner.

On the other hand, two ultrafilters $\mathcal{U}, V$ are said to be contiguous if for every $X \in \mathcal{U}, Y \in \mathcal{V}$ the distance from $X$ to $Y$ is null. Then it is easily checked that if $\mathcal{U}, \mathcal{V}$ are contiguous coroner ultrafilters, then $\mathcal{J}(\mathcal{U})=\mathcal{J}(\mathcal{V})$. An interesting question is whether two coroner ultrafilters $\mathcal{U}, \mathcal{V}$, defining maximal ideals, such that $\mathcal{J}(\mathcal{U})=\mathcal{J}(\mathcal{V})$, are contiguous. In [11], it is proved that, as far as uniformly continuous functions are concerned, two ultrafilters define the same maximal ideal if and only if they are contiguous.

The following Proposition is easily checked:

Proposition : Let $\left(a_{n}\right)_{n \in \mathbb{N}}$ be a coroner sequence. Both families 
$X_{q}=\bigcup_{n=q}^{\infty} \Gamma\left(a_{n},, \rho_{n},\left|a_{n}\right|\right), 0<\rho_{n}<\left|a_{n}\right|, q \in \mathbb{N}$ and $S_{q}=\bigcup_{n=q}^{\infty} \Gamma\left(a_{n}, r, \rho_{n}\right), 0<r<\rho_{n}<\left|a_{n}\right|, q \in \mathbb{N}, \lim _{n \rightarrow \infty} \rho_{n}=1$ are basis of coroner filters.

Definitions and notation: Let $\left(a_{n}\right)_{n \in \mathbb{N}}$ be a coroner sequence. We will denote by $\mathcal{X}\left(a_{n}\right)$ the filter admitting for basis the family $X_{q}=\bigcup_{n=q}^{\infty} \Gamma\left(a_{n},, \rho_{n},\left|a_{n}\right|\right), 0<$ $\rho_{n}<\left|a_{n}\right|, q \in \mathbb{N}$ and by $\mathcal{S}\left(a_{n}\right)$ the filter admitting for basis the family

$$
S_{q}=\bigcup_{n=q}^{\infty} \Gamma\left(a_{n}, r, \rho_{n}\right), 0<r<\rho_{n}<\left|a_{n}\right|, q \in \mathbb{N}
$$

A coroner ultrafilter $\mathcal{U}$ will be said to follow the sequence $\left(a_{n}\right)_{n \in \mathbb{N}}$ if it is secant with the filter generated by the family $T_{q}=\bigcup_{n=q}^{\infty} d\left(a_{n},\left|a_{n}\right|^{-}\right), q \in \mathbb{N}$.

A coroner ultrafilter $\mathcal{U}$ following a sequence $\left(a_{n}\right)_{n \in \mathbb{N}}$ will be said to be slim if it is secant with a set $\bigcup_{n=0}^{\infty} d\left(a_{n}, r^{-}\right)$for some $\left.r \in\right] 0,1[$.

It will be said to be wide if it is thinner than the filter $\mathcal{X}\left(a_{n}\right)$ and it will be said to be intermediate if it is thinner than the filter $\mathcal{S}\left(a_{n}\right)$.

Recall that a coroner sequence $\left(a_{n}\right)_{n \in \mathbb{N}}$ is said to be regular or to be an interpolation sequence if $\inf _{j \in \mathbb{N}} \prod_{i \neq j}\left|a_{i}-a_{j}\right|>0$ [7], [14]. A coroner ultrafilter $\mathcal{U}$ is said to be regular if it is thinner than a regular sequence.

Theorem 1: $\quad$ Let $\mathcal{U}$ be a coroner ultrafilter that is not thinner than $\mathcal{Y}$. Then it follows a coroner sequence $\left(a_{n}\right)_{n \in \mathbb{N}}$. Moreover, either it is slim, or it is wide, or it is intermediate.

Theorem 2: $\quad$ Let $\mathcal{U}$ be an ultrafilter thinner than $\mathcal{Y}$. Then $\mathcal{J}(\mathcal{U})=\{0\}$.

Theorem 3: $\quad$ Let $\mathcal{U}$ be a wide ultrafilter. Then $\mathcal{J}(\mathcal{U})=\{0\}$.

We must recall Theorem A [7]:

Theorem A: Let $\mathcal{U}$ be a regular ultrafilter. Then $\mathcal{J}(\mathcal{U})$ is a maximal ideal of $A$.

Here we can give a precision:

Theorem 4: Suppose $K$ is spherically complete. Let $\mathcal{U}$ be a regular coroner ultrafilter. Then $\mathcal{J}(\mathcal{U})$ is a maximal ideal that is not differential.

Now we can state Theorem 5:

Theorem 5: Let $\left(a_{n}\right)_{n \in \mathbb{N}}$ be a coroner sequence such that $\prod_{n=0}^{\infty}\left|a_{n}\right|>0$, let $r \in$ ]0,1[ and let $h \in A$. If $\lim _{n \rightarrow \infty}\|h\|_{d\left(a_{n}, r\right)}=0$, then $\lim _{n \rightarrow \infty}\left\|h^{(k)}\right\|_{d\left(a_{n}, r\right)}=0 \quad \forall k \in \mathbb{N}$. 
Theorem 6: Let $r \in] 0,1[$ and let $\mathcal{U}$ be a slim coroner ultrafilter secant with a coroner sequence $d\left(a_{n}, r\right)$ such that $\prod_{n=0}^{\infty}\left|a_{n}\right|>0$ and let $h \in A$. Then the following two statements are equivalent:

1) $h^{(k)} \in \mathcal{J}(\mathcal{U}) \forall k \in \mathbb{N}$,

2) $\mathcal{U}$ is secant with a subsequence $d\left(a_{s(m)}, r\right)$ of the sequence $\left(d\left(a_{n}, r\right)\right)_{n \in \mathbb{N}}$ such that

$\lim _{m \rightarrow \infty}\|h\|_{d\left(a_{s(m)}, r\right)}=0$.

Moreover, if 1), 2) are satisfied, then $\left.\lim _{m \rightarrow \infty}\|h\|_{d\left(a_{s(m)}, \rho\right)}=0 \forall \rho \in\right] 0,1[$.

Corollary 6.1: Let $\left(a_{n}\right)_{n \in \mathbb{N}}$ be a coroner sequence such that $\prod_{n=0}^{\infty}\left|a_{n}\right|>0$, let $r \in] 0,1\left[\right.$ and let $h \in A$. If $\lim _{n \rightarrow \infty}\|h\|_{d\left(a_{n}, r\right)}=0$, then $\left.\lim _{m \rightarrow \infty}\|h\|_{d\left(a_{n}, \rho\right)}=0 \forall \rho \in\right] 0,1[$.

Theorem 7: Suppose $K$ is spherically complete. Let $\mathcal{U}$ be a coroner ultrafilter. If $\mathcal{J}(\mathcal{U})$ is not differential, then it is a maximal ideal of $A$.

Theorem 8: Suppose $K$ is spherically complete. Let $\mathcal{U}$ be a slim coroner ultrafilter. Then $\mathcal{J}(\mathcal{U})$ is a maximal ideal that is not differential.

Theorem 9: $\quad$ Suppose $K$ is spherically complete. Let $\mathcal{U}, V$ be coroner ultrafilters such that $\mathcal{J}(\mathcal{U})$ is not differential. Then $\mathcal{J}(\mathcal{U})=\mathcal{J}(\mathcal{V})$ if and only if $\mathcal{U}$ and $\mathcal{V}$ are contiguous.

Corollary 9.1: Suppose $K$ is spherically complete. Let $\mathcal{U}, V$ be coroner ultrafilters, with $\mathcal{U}$ slim. Then $\mathcal{J}(\mathcal{U})=\mathcal{J}(\mathcal{V})$ if and only if $\mathcal{U}$ and $\mathcal{V}$ are contiguous.

Concerning continuous multiplicative semi-norms whose kernel is neither zero nor a maximal ideal, J. Araujo showed their existence [1]. From [1] we can extract the following result:

Theorem 10 (J. Araujo): Let $\left(\alpha_{n}\right)_{n \in \mathbb{N}}$ be a coroner sequence together with a sequence of integers $\left(q_{n}\right)_{n \in \mathbb{N}}$ such that $\lim _{n \rightarrow+\infty} q_{n}=+\infty$ and $\prod_{n=1}^{\infty}\left|\alpha_{n}\right|^{q_{n}}>0$. Let $\mathcal{H}$ be an ultrafilter on $\mathbb{N}$. For each $r \in] 0,1[$, let $\mathcal{P}(\mathcal{H})$ be the ideal of the $f \in A$ such that $\lim _{\mathcal{H}}\|f\|_{d\left(\alpha_{n}, r\right)}=0$. Then $\mathcal{P}(\mathcal{H})$ is differential and does not depend on $r$, is a non-zero prime closed ideal and is the kernel of elements $\phi_{r} \in \operatorname{Mult}(A, \|$. $\|)$ defined as $\phi_{r}(f)=\lim _{\mathcal{U}}\|f\|_{d\left(\alpha_{n}, r\right)}$ and $\phi(r)$ lies in the closure of $\operatorname{Mult}_{a}(A, \|$. $\|)$. Moreover, if the sequence $\left(\alpha_{n}\right)_{n \in \mathbb{N}}$ satisfies $\lim _{n \rightarrow+\infty}\left|\alpha_{n}\right|^{q_{n+1}}=0$, then $\mathcal{P}$ is not a maximal ideal and the set $\left\{\phi_{r} \mid r \in\right] 0,1[\}$ is homeomorphic to $] 0,1[$ through the mapping $\Psi$ defined by $\Psi(r)=\phi_{r}$.

Example: We can choose $\alpha_{n}$ such that $-\log \left(\left|\alpha_{n}\right|\right) \in\left[\frac{1}{n^{3}}, \frac{2}{n^{3}}\right]$ with $q_{n}=n$.

Unsolved questions: 1) Do exist differential maximal ideals in $A$ ? Clearly, when $K$ is spherically complete, such an ideal should be of the form $\mathcal{J}(\mathcal{U})$ with $\mathcal{U}$ an 
intermediate coroner ultrafilter. Thus, the first question is: given an intermediate ultrafilter $\mathcal{U}$, is $\mathcal{J}(\mathcal{U})$ a maximal ideal?

2) Can an intermediate ultrafilter be regular? Clearly, we can define a regular sequence $\left(a_{n}\right)_{n \in \mathbb{N}}$ such that, for example, $\left|a_{2 n}\right|\left(\frac{n-1}{n}\right)<\left|a_{2 n}-a_{2 n+1}\right|<\left|a_{2 n}\right|$. But does exist an intermediate ultrafilter thinner than that sequence? 3) Do exist continuous multiplicative semi-norms whose kernel is non-zero, non-maximal ideal other than those of Araujo's type? If the field is spherically complete, by [9] we know that all continuous multiplicative semi-norms whose kernel is either zero or a maximal ideal, lie in the closure of $\operatorname{Mult}_{a}(A,\|\|$.$) . And as it was noticed, so do$ Araujo's type semi-norms.

Clearly, if we could prove that all continuous multiplicative semi-norms are defined by filters on $D$ (which is the case for all those already known), then $\operatorname{Mult}_{a}(A,\|\|$.$) would be dense in the whole set \operatorname{Mult}(A,\|\|$.$) .$

On the other hand, each time we use the hypothesis $K$ spherically complete this is just to split certain function in a product of two factors: one having zeroes in a certain subset of $D$ and the other in the complemental, because such a factorization requires Lazard's Theorem. But such an hypothesis seems unlikely linked to the results.

\section{The proofs}

Notation: Let $X \subset K$ be different from $K$. We denote by $\operatorname{codiam}(X)$ the distance from $X$ to $K \backslash X$. Let $f \in A$. For every $r \in] 0,1[$ and let $\mathcal{F}(r)$ be the circular filter of center 0 and diameter $r$. We set $|f|(r)=\lim _{\mathcal{F}(r)}|f(x)|$.

Proof of Theorem 1: Suppose $\mathcal{U}$ is not secant with $\mathcal{Y}$ and let $X \in \mathcal{Y}$ be such that $\mathcal{U}$ is not secant with $X$. By definition of $\mathcal{Y}, X$ contains a set of the form

$D \backslash\left(\bigcup_{j=1}^{\infty} d\left(a_{n},\left|a_{n}\right|^{-}\right)\right)$, with $\left(a_{n}\right)_{n \in \mathbb{N}}$ a coroner sequence. Consequently, $\mathcal{U}$ admits an element included in $\bigcup_{j=1}^{\infty} d\left(a_{n},\left|a_{n}\right|^{-}\right)$and therefore it follows the sequence $\left(a_{n}\right)_{n \in \mathbb{N}}$.

Now, suppose $\mathcal{U}$ is not slim. There exists a sequence $\left(\rho_{n}\right)_{n \in \mathbb{N}}$ with $0<\rho_{n}<$ $\left|a_{n}\right|, n \in \mathbb{N}$ and $\lim _{n \rightarrow+\infty} \rho_{n}=1$ such that $\mathcal{U}$ is secant with $\bigcup_{n=0} \Gamma\left(a_{n}, \rho_{n},\left|a_{n}\right|\right)$. Suppose now it is not intermediate either. Then for every sequence $\left(r_{n}\right)$ with $0<\rho_{n}<$ $r_{n}, \mathcal{U}$ is not secant with $\bigcup_{n=0} \Gamma\left(a_{n}, \rho_{n}, r_{n}\right)$ hence it is secant with $\bigcup_{n=0} \Gamma\left(a_{n}, r_{n},\left|a_{n}\right|\right)$ which ends the proof.

Proof of Theorem 2: Let $\left(a_{n}\right)_{n \in \mathbb{N}}$ be a coroner sequence and consider the family $\mathcal{H}$ of all sets of the form $\bigcup_{j=q}^{\infty} \Gamma\left(a_{n}, r_{n},\left|a_{n}\right|\right)$ with $0<r_{n}<\left|a_{n}\right|$. Clearly $\mathcal{H}$ is a basis of a filter $\mathcal{F}$. Now, let $\mathcal{U}$ be an ultrafilter thinner than $\mathcal{F}$. Then $\mathcal{U}$ follows the sequence $\left(a_{n}\right)_{n \in \mathbb{N}}$. We will check that $\mathcal{U}$ is not secant with any coroner sequence $d\left(b_{m}, r\right)_{m \in \mathbb{N}}$. Indeed, suppose that $\mathcal{U}$ is secant with a coroner sequence $d\left(b_{m}, r\right)_{m \in \mathbb{N}}$. Without loss of generality, we can assume that $\left|b_{m}\right|>$ 
$r \forall m \in \mathbb{N}$. By hypothesis, $\mathcal{U}$ is secant with a set $S=\bigcup_{j=q}^{\infty} \Gamma\left(a_{n}, r_{n},\left|a_{n}\right|\right)$. hence each $b_{m}$ must belong to a disk $d\left(a_{s(m)},\left|a_{s(m)}\right|^{-}\right)$, so that $\left|b_{m}-a_{s(m)}\right|<\left|a_{s(m)}\right|$. For each $m \in \mathbb{N}$, let $u_{m}=\max \left(r,\left|b_{m}-a_{s(m)}\right|\right)$ and let $\left.r_{m} \in\right] u_{m},\left|a_{m}\right|[$. Then the set $X=D \backslash \bigcup_{j=1}^{\infty} d\left(a_{s(m)}, r_{m}\right)$ belongs to $\mathcal{F}$ but each disk $d\left(b_{m}, r\right)$ has empty intersection with $X$. Consequently, $\mathcal{U}$ can't be secant with any coroner sequence $d\left(b_{m}, r\right)_{m \in \mathbb{N}}$ and therefore is a wide ultrafilter.

Proof of Theorem 2: Let $f \in \mathcal{J}(\mathcal{U})$ and suppose $f \neq 0$. Let $\left(d\left(a_{n},\left|a_{n}\right|^{-}\right)_{n \in \mathbb{N}}\right)$ be the sequence of classes containing zeroes of $f$ and let $S=D \backslash\left(\bigcup_{n=0}^{\infty} d\left(a_{n},\left|a_{n}\right|^{-}\right)\right)$. Then by hypothesis $\mathcal{U}$ is secant with $S$. Now, let $u \in S$ and let $r=|u|$. Since $f$ has no zero in $d\left(u, r^{-}\right)$, it satisfies $|f(u)|=|f|(r)$. Consequently, $\lim _{\mathcal{U}}|f(x)|=$ $\lim _{r \rightarrow 1}|f|(r) \mid=\|f\|$, a contradiction.

Proof of Theorem 3: $\quad$ Let $f \in \mathcal{J}(\mathcal{U})$ and suppose $f \neq 0$. Let $\left(d\left(a_{n},\left|a_{n}\right|^{-}\right)-{ }_{n \in \mathbb{N}}\right.$ be the coroner sequence of disks containing all zeroes of $f$. For each $n \in \mathbb{N}$, set $r_{n}=$ $\left|a_{n}\right|$. We can notice that for every $x \notin \bigcup_{n=0}^{\infty} d\left(a_{n}, r_{n}^{-}\right)$, we have $|f(x)|=|f|(|x|)$. Consequently, since $\lim _{\mathcal{U}} f(x)=0, \mathcal{U}$ must follow the sequence $\left(a_{n}\right)_{n \in \mathbb{N}}$. So, for each $n \in$ $\mathbb{N}$, there exists $\left.\rho_{n} \in\right] 0, r_{n}\left[\right.$ such that $|f(x)|>\left(\frac{n-1}{n}\right)|f|\left(r_{n}\right) \forall x \in \Gamma\left(a_{n}, \rho_{n}, r_{n}\right)$. Now, let us fix $q \in \mathbb{N}^{*}$ and let $X_{q}=\bigcup_{n=q}^{\infty} \Gamma\left(a_{n}, \rho_{n}, r_{n}\right)$. On the one hand, $X_{q}$ belongs to $\mathcal{U}$. On the other hand, we just saw that $|f(x)|>\left(\frac{n-1}{n}\right)|f|\left(r_{n}\right) \forall x \in \Gamma\left(a_{n}, \rho_{n}, r_{n}\right)$ which shows that $\lim _{\mathcal{U}}|f(x)|=\|f\|$, a contradiction to the hypothesis $f \in \mathcal{J}(\mathcal{U})$.

Lemmas 1, 2, 3 are immediate:

Lemma 1: Let $E$ be a disk and $X$ be a closed open subset of $E$. Then $\operatorname{codiam}(X)=$ $\operatorname{codiam}(E \backslash X)$.

Lemma 2: Let $X_{1}, \ldots, X_{n}$ be closed open subsets of $K$ such that $\bigcap_{j=1}^{n} X_{n} \neq \emptyset$ and such that $\operatorname{codiam}\left(X_{j}\right)>0$. Then $\operatorname{codiam}\left(\bigcap_{j=1}^{n} X_{n}\right)>0$.

Lemma 3: Let $f_{1}, \ldots, f_{n} \in A$, let $\left.m_{1}, \ldots, m_{n} \in\right] 0,+\infty\left[\right.$ and let $\Delta=\left\{x \in D|| f_{j}(x) \mid \geq\right.$ $\left.m_{j}, j=1, \ldots, n\right\}$. Then $\operatorname{codiam}(\Delta)>0$.

Lemmas 4, 5, 6 are classical and may be found in [5]:

Lemma 4: Let $f \in A$, let $d\left(a, r^{-}\right) \subset U$ and let $m=\varphi_{a, r}\left(f^{\prime}\right)$. Then $\|f\|_{d\left(a, r^{-}\right)} \geq$ $m r$. Moreover, if $f$ has no zero in $d\left(a, r^{-}\right)$, then $|f(x)|$ is a constant $c \geq m r$ in all of $d\left(a, r^{-}\right)$. 
Lemma 5: Let $f \in A$, let $a \in U$ and let $r \in] 0,1\left[\right.$. For every $k \in \mathbb{N}^{*}$, we have $\left\|f^{(k)}\right\|_{d\left(a, r^{-}\right)} \leq \frac{\|f\|_{d\left(a, r^{-}\right)}}{r^{k}}$.

Lemma 6: Let $a \in D$ and let $r, s \in] 0,1]$ be such that $r<s$ and let $f, g \in A$ be such that every zero of $f$ order $t$ is a zero of $g$ of order $u \geq t$. Then $g$ factorizes in $A$ in the form $g=f h$ with $h \in A$ and $f, g$ satisfy

$$
\frac{\|f\|_{d(a, s)}}{\|f\|_{d(a, r)}} \leq \frac{\|g\|_{d(a, s)}}{\|g\|_{d(a, r)}}
$$

Lemma 7 is proved in [14]:

Lemma 7: Let $f_{1}, \ldots, f_{q} \in A$ satisfy $\left\|f_{j}\right\|<1 \forall j=1, \ldots, q$ and $\inf \left\{\max _{j=1, \ldots, q}\left(\left|f_{j}(x)\right|\right) \mid x \in D\right\}=\omega>0$. There exist $g_{1}, \ldots, g_{q} \in$ A such that $\sum_{j=1}^{q} g_{j} f_{j}=1$ and $\max _{j=1, \ldots, q}\left\|g_{j}\right\|<\omega^{-2}$.

Lemma 8: Let $\mathcal{U}$ be a coroner ultrafilter, $f \in \mathcal{J}(\mathcal{U})$ and let $\Lambda \in \mathcal{U}$ have codiameter $\rho>0$. If $\inf _{x \in \Lambda}\left|f^{\prime}(x)\right|>0$, then $f$ admits zeroes in $\Lambda$.

Proof: Suppose $f$ has no zero in $\Lambda$. Let $\inf _{x \in \Lambda}\left|f^{\prime}(x)\right|$ and let $a \in \Lambda$. Then $d\left(a, \rho^{-}\right) \subset \Lambda$. In $d\left(a, \rho^{-}\right), f(x)$ is of the form $\sum_{n=0}^{\infty} \alpha_{n}(x-a)^{n}$, with $\left|a_{1}\right| \geq l$, hence $\|f\|_{d\left(a, \rho^{-}\right)} \geq l \rho$. But since $f$ has no zero in $d\left(a, \rho^{-}\right),|f(a)|=\|f\|_{d\left(a, \rho^{-}\right)}$, hence $|f(a)| \geq l \rho$, a contradiction to the hypothesis $f \in \mathcal{J}(\mathcal{U})$.

Proof of Theorem 4: Since $\mathcal{U}$ is regular, by Theorem A we know that $\mathcal{J}(\mathcal{U})$ is a maximal ideal. Let $\left.\left(a_{n}\right)_{n \in \mathbb{N}}\right)$ be a regular sequence of $D$ such that $\mathcal{U}$ is thinner than this sequence.

Since $K$ is spherically complete, there exists $f \in A$ admitting each $a_{n}$ as a zero of order 1 and admitting no other zero. Then by Corollary 4.8 [14], the ideal generated by $f$ and $f^{\prime}$ is $A$, hence $f^{\prime}$ may not belong to $\mathcal{J}(\mathcal{U})$.

Proof of Theorem 5: Suppose that $\lim _{n \rightarrow \infty}\|h\|_{d\left(a_{n}, r\right)}=0$. By Lemma 5, we have $\left\|h^{(k)}\right\|_{d\left(a_{n}, r\right)} \leq\|h\|_{\frac{d\left(a_{n}, r\right)}{r^{k}}}$ and hence $\lim _{n \rightarrow \infty}\left\|h^{(k)}\right\|_{d\left(a_{n}, r\right)}=0$.

Proof of Theorem 6: Suppose first that 2) holds: $\mathcal{U}$ is secant with a subsequence $\left(d\left(a_{s(m)}, r\right)\right.$ of the sequence $\left(d\left(a_{n}, r\right)\right)$ such that $\lim _{m \rightarrow \infty}\|h\|_{d\left(a_{s(m)}, r\right)}=0$. Let us consider $h^{(k)}$. By Lemma 5, we have $\left\|h^{(k)}\right\|_{d\left(a_{s(m)}, r\right)} \leq\|h\|_{\frac{d\left(a_{s(m)}, r\right)}{r^{k}}}$ and hence $\lim _{m \rightarrow \infty}\left\|h^{(k)}\right\|_{d\left(a_{s(m)}, r\right)}=0$. For each $m \in \mathbb{N}^{*}$, set $\epsilon_{m}=\left\|h^{(k)}\right\|_{d\left(a_{s(m)}, r\right)}$.

Since $\mathcal{U}$ is secant with the sequence $\left(d\left(a_{s(m)}, r\right)\right)$ and since $\mathcal{U}$ is an ultrafilter, there exists $X_{m} \in \mathcal{U}$ such that $X_{m} \subset \bigcup_{j=m}^{\infty} d\left(a_{s(j)}, r\right)$ and hence $\left|h^{(k)}(x)\right| \leq \epsilon_{m} \forall x \in$ $X_{m}$, which proves that $h^{(k)}$ belongs to $\mathcal{J}(\mathcal{U})$. 
Suppose now that 1$)$ holds and let $\rho \in[r, 1[$. We will prove that 2) is satisfied and for this it sufficient to show that $\left.\lim _{m \rightarrow \infty}\|h\|_{d\left(a_{s(m)}, \rho\right)}=0 \forall \rho \in\right] 0,1[$.

For every $m \in \mathbb{N}$, we can take $q_{m} \in \mathbb{N}$ such that $\rho^{q_{m}}<\frac{1}{m}$. Suppose we have defined integers $s(l)$ up to $l=m-1$ satisfying $\|h\|_{d\left(a_{s(l)}, \rho\right)} \leq \frac{1}{l}, 1 \leq l \leq m-1$. Let us define $s(m)$ in the same way. Let $X \in \mathcal{U}$ be such that $\left\|h^{(k)}\right\|_{X} \leq \frac{|k !|}{m} \forall k=0, \ldots, q_{m}$. Since $\mathcal{U}$ is secant with the sequence $\left(d\left(a_{n}, r\right)\right)$, there exists $s(m) \in \mathbb{N}, s(m)>$ $s(m-1)$ such that $\left|a_{s(m)}\right|>\left|a_{s(m-1)}\right|$ and $a_{s(m)} \in X$. Then we have $\left|h^{(k)}\left(a_{s(m)}\right)\right|<\frac{|k !|}{m} \forall k \leq q_{m}$.

Now, consider $\|h\|_{d\left(a_{s(m)}, \rho\right)}$. Taking the point $a_{s(m)}$ as origin and putting $x=$ $a_{s(m)}+u, h(x)$ has expansion in the form $\sum_{j=0}^{\infty} \alpha_{j} u^{j}$ with $\left|\alpha_{j}\right|=\frac{\left|h^{(j)}\left(a_{s(m)}\right)\right|}{|j !|} \forall j \leq q_{m}$. Consequently, $\left|\alpha_{j}\right| \rho^{j} \leq \frac{1}{m} \forall j \leq q_{m}$.

Next, as $\|h\| \leq 1$, each $\left|\alpha_{j}\right|$ is $\leq 1$. Hence, taking into account that $\rho^{q_{m}} \leq$ $\frac{1}{m}$, we have $\left|\alpha_{j}\right| \rho^{j} \leq \frac{1}{m} \forall j \in \mathbb{N}$ and therefore $\left|\sum_{j=0}^{\infty} \alpha_{j} u^{j}\right| \leq \frac{1}{m} \forall u \in d(0, \rho)$ i.e. $|h(x)| \leq \frac{1}{m} \forall x \in d\left(a_{s(m)}, \rho\right)$. Thus $\|h\|_{d\left(a_{s(m)}, \rho\right)} \leq \frac{1}{m} \forall m \in \mathbb{N}$. So much the more, $\left.\left.\|h\|_{d\left(a_{s(m)}, \rho\right)} \leq \frac{1}{m} \forall m \in \mathbb{N}, \forall \rho \in\right] 0, r\right]$. This ends the proof of Theorem 6 .

Proof of Theorem 7: Suppose $\mathcal{J}(\mathcal{U})$ is not differential. Then there exists $h \in$ $\mathcal{J}(\mathcal{U})$ such that $h^{\prime} \notin \mathcal{J}(\mathcal{U})$. Let $f \in A \backslash \mathcal{J}(\mathcal{U})$ and let us show that $f A+\mathcal{J}(\mathcal{U})=A$.

Since $f \notin \mathcal{J}(\mathcal{U})$ and since $h^{\prime} \notin \mathcal{J}(\mathcal{U})$ we have $\varphi_{\mathcal{U}}(f) \varphi\left(h^{\prime}\right) \neq 0$. Let $\varphi_{\mathcal{U}}(f)=m$ and $\varphi_{\mathcal{U}}\left(h^{\prime}\right)=l$. Let $M=\{x \in D|| f(x) \mid \geq m\}, L=\left\{x \in D|| h^{\prime}(x) \mid \geq l\right\}$ and let $\Lambda=L \cap M$. Let $\rho=\operatorname{codiam}(\Lambda)$ and let $\Theta=D \backslash \Lambda$. Then by Lemma 3, $\rho>0$ and $\operatorname{codiam}(\Theta)=\rho$.

Since $K$ is spherically complete, by [13] there exist $\widetilde{h}, \widehat{h} \in A$ such that $h=\widehat{h} \widetilde{h}$, with $\widetilde{h}(x) \neq 0 \forall x \in \Lambda$ and $\widehat{h}(x) \neq 0 \forall x \in \Theta$.

So we have

(1) $h^{\prime}=(\widetilde{h})^{\prime} \widehat{h}+\widetilde{h}(\widehat{h})^{\prime}$.

Let $a \in \Theta^{\prime}$ and let $\sigma$ be the distance from $a$ to $\Lambda$. By construction, each zero of $\widehat{h}$ of order $q$ is a zero of $h$ with an order $u \geq q$. Consequently, by Lemma 6 we have

$$
\frac{\|\widehat{h}\|_{d\left(a, \sigma^{-}\right)}}{\|\widehat{h}\|} \geq \frac{\|h\|_{d\left(a, \sigma^{-}\right)}}{\|h\|}
$$

Setting $\mu=\frac{\|\widehat{h}\|}{\|h\|}$, we derive

$$
\|\widehat{h}\|_{d\left(a, \sigma^{-}\right)} \geq \mu\|h\|_{d\left(a, \sigma^{-}\right)} .
$$

And since $\widehat{h}$ has no zero in $\Theta$, we have $|\widehat{h}(a)|=\|\widehat{h}\|_{d\left(a, \sigma^{-}\right)}$. Now, the distance from $a$ to $L$ is a number $t \geq \sigma \geq \rho$. Consequently, $\varphi_{a, t}\left(h^{\prime}\right) \geq \varphi_{a, \sigma}\left(h^{\prime}\right) \geq l$, hence 
$\left\|h^{\prime}\right\|_{d(a, t)} \geq l$ and therefore, by Lemma 4

$$
\|h\|_{d(a, t)} \geq l t \geq l \rho .
$$

Now, by (2) and (3), we derive $|\widehat{h}(a)| \geq \mu l \rho$. This proves that $\min (|f(x)|,|\widehat{h}(x)|) \geq$ $\min (m, \mu l \rho) \forall x \in D$ and therefore by Lemma $7, f A+\mathcal{J}(\mathcal{U})=A$.

Now, it only remains us to prove that $\widehat{h}$ belongs to $\mathcal{J}(\mathcal{U})$. We will first prove that $\widetilde{h} \notin \mathcal{J}(\mathcal{U})$. Indeed, suppose $\widetilde{h} \in \mathcal{J}(\mathcal{U})$. By $(1)$ we see that $(\widetilde{h})^{\prime} \notin \mathcal{J}(\mathcal{U})$. Let $s=\varphi_{\mathcal{U}}\left((\widetilde{h})^{\prime}\right)$, let $\Xi=\left\{x \in \Lambda||(\widetilde{h})^{\prime}(x) \mid=s\right\}$ and let $\tau=\operatorname{codiam}(\Xi)$. By Lemma $2, \tau>0$. But since $\Xi$ is included in $\Lambda, \widetilde{h}$ has no zero in that $\Xi$, a contradiction by Lemma 8 . Thus, $\widetilde{h} \notin \mathcal{J}(\mathcal{U})$ and therefore, as $\mathcal{J}(\mathcal{U})$ is prime, $\widehat{h}$ does belong to $\mathcal{J}(\mathcal{U})$, which ends the proof.

Lemma 9: Let $r \in] 0,1\left[\right.$ and let $f \in A$ have a unique zero in $d\left(0, r^{-}\right)$. Then, $f^{\prime}$ has no zero in $d\left(0, r^{-}\right)$.

Proof : Without loss of generality, we may assume that 0 is the unique zero of $f$ in $d\left(0, r^{-}\right)$. So, in $d\left(0, r^{-}\right), f(x)$ has expansion in the form $\sum_{n=1}^{\infty} a_{n} x^{n}$ with $\left|a_{n}\right| r^{n-1}<\left|a_{1}\right| \forall n>1$. Consequently, $f^{\prime}(x)=\left|a_{1}\right| \forall x \in d\left(0, r^{-}\right)$.

Proof of Theorem 8: Since $\mathcal{U}$ is slim, there exists a sequence $\left(a_{n}\right)_{n \in \mathbb{N}}$ with $\lim _{n \rightarrow+\infty}\left|a_{n}\right|=1, \prod_{n=0}^{\infty}\left|a_{n}\right|>0, r>0$, such that $\mathcal{U}$ is secant with the sequence $\left(d\left(a_{n}, r\right)\right)_{n \in \mathbb{N}}$.

Since $K$ is spherically complete and since $\prod_{n=0}^{\infty}\left|a_{n}\right|>0$, there exists $f \in A$ admitting each $a_{n}$ as a zero of order 1 and admitting no other zero. Thus, by Lemma 9 , in each disk $d\left(a_{n}, r_{n}^{-}\right), f^{\prime}$ has no zero and therefore

(1) $\left|f^{\prime}(x)\right|=\left|f^{\prime}\right|\left(r_{m}\right) \forall x \in d\left(a_{n}, r_{n}^{-}\right)$.

Particularly, (1) holds in $d\left(b_{m}, r\right)$ and therefore $f^{\prime}(x)$ does not tend to 0 on $\mathcal{U}$.

Now, suppose $\mathcal{J}(\mathcal{U})$ is differential. Let $X \in \mathcal{U}$ be included in $\bigcup_{n=0}^{\infty} d\left(a_{n}, r\right)$. For convenience, set $r_{n}=\left|a_{n}\right|$. Let $h \in \mathcal{J}(\mathcal{U})$. Since $X \in \mathcal{U} \subset \bigcup_{n=0}^{\infty} d\left(a_{n}, r\right)$, there exists a sequence of integers $\left(s(m)_{m \in \mathbb{N}}\right)$ and a sequence $\left(b_{m}\right)_{m \in \mathbb{N}}$ with $b_{m} \in d\left(a_{s(m)}, r\right)$ such that $\lim _{m \rightarrow \infty} h\left(b_{m}\right)=\lim _{m \rightarrow \infty} h^{\prime}\left(b_{m}\right)=0$. But by $(1), f$ does not belong to $\mathcal{J}(\mathcal{U})$ and therefore, the ideal $\mathcal{H}=f A+\mathcal{J}(\mathcal{U})$ strictly contains $\mathcal{J}(\mathcal{U})$.

On the other hand, suppose $\mathcal{H}=A$ and let $1=u f+g \in \mathcal{H}$ with $u \in A$ and $g \in \mathcal{J}(\mathcal{U})$. Then, at a point $a_{m}$ we have $1 \leq\|g\|_{d\left(a_{m}, r^{-}\right)}$. But since $\mathcal{U}$ is secant with the sequence $\left(d\left(a_{m}, r\right)\right)$ and since $\mathcal{U}$ is differentiable, by Theorem 6 we have $\liminf _{n \rightarrow \infty}\|g\|_{d\left(a_{m}, r^{-}\right)}=0$, a contradiction.

Consequently, $\mathcal{H} \neq A$ and this finishes proving that if $\mathcal{J}(\mathcal{U})$ is differential, it is not a maximal ideal.

In [9] we proved the following result: 
Lemma 10: Let $K$ be spherically complete and let $\mathcal{U}$ be a coroner ultrafilter. Let $E \in \mathcal{U}$ and let $h \in A$ be in the form $\bar{h} \widehat{h}$ where $\bar{h}, \widehat{h} \in A$ are such that the zeroes of $\widehat{h}$ are the zeroes of $h$ in $E$ and the zeroes of $\bar{h}$ are the zeroes of $h$ in $D \backslash E$. Then $\widehat{h}$ belongs to $\mathcal{J}(\mathcal{U})$ and $(\widehat{h})^{\prime}$ does not belong to $\mathcal{J}(\mathcal{U})$.

Proof of Theorem 9: If $\mathcal{U}$ and $\mathcal{V}$ are contiguous, we know that $\mathcal{J}(\mathcal{U})=\mathcal{J}(\mathcal{V})$ due to the fact that functions of $A$ are uniformly continuous [7].

Now, suppose that $\mathcal{J}(\mathcal{U})=\mathcal{J}(\mathcal{V})$ and that $\mathcal{U}$ and $\mathcal{V}$ are not contiguous. Let $r=\delta(\mathcal{U}, \mathcal{V})$. We then can find $E \in \mathcal{U}$ with a strictly positive codiameter and $F \in \mathcal{V}$ such that $\delta(E, F)>\frac{2 r}{3}$. Since $\mathcal{U}$ is not differential, there exists $h \in \mathcal{J}(\mathcal{U})$ such that $h^{\prime} \notin \mathcal{J}(\mathcal{U})$. Since $K$ is spherically complete, we can factorize $h$ in the form $\widehat{h} \widehat{h}$ where $\bar{h}, \widehat{h} \in A$ are such that the zeroes of $\widehat{h}$ are the zeroes of $h$ in $E$ and the zeroes of $\bar{h}$ are the zeroes of $h$ in $D \backslash E$. Then by Lemma A $\widehat{h}$ belongs to $\mathcal{J}(\mathcal{U})$ and $(\widehat{h})^{\prime}$ does not belong to $\mathcal{J}(\mathcal{U})$.

Suppose first that we can find $E \in \mathcal{U}$ and $F \in \mathcal{V}$ defined as above, such that $\inf _{x \in F}|\widehat{h}(x)|>0$. Then $\lim _{\mathcal{V}}|\widehat{h}(x)|>0$ and therefore $\widehat{h}$ does not belong to $\mathcal{J}(\mathcal{V})$, a contradiction to the hypothesis.

Suppose now that we can't find $E \in \mathcal{U}$ and $F \in \mathcal{V}$ defined as above, such that $\inf _{x \in F}|\widehat{h}(x)|>0$. So, for every $E \in \mathcal{U}$ and $F \in \mathcal{V}$ such that $\delta(E, F)>\frac{2 r}{3}$, we have $\inf _{x \in F}|\widehat{h}(x)|=0$. Let us fix $E \in \mathcal{U}$ and $F \in \mathcal{V}$ defined as above, such that $\delta(E, F)>\frac{2 r}{3}$ and let $s=\frac{2 r}{3}$.

Since $\mathcal{J}(\mathcal{U})=\mathcal{J}(\mathcal{V}), \widehat{h}$ belongs to $\mathcal{J}(\mathcal{V})$. Take $\epsilon>0$. There exists $b \in F$ such that $|\widehat{h}(b)|<\epsilon$. Now we have $d\left(b, s^{-}\right) \cap E=\emptyset$. Consequently, $\widehat{h}$ has no zero in $d\left(b, s^{-}\right)$and therefore $\|\widehat{h}\|_{d\left(b, s^{-}\right)}<\epsilon$. Then by Lemma $5\left\|\widehat{h}^{(k)}\right\|_{d\left(b, s^{-}\right)}<\frac{\epsilon}{s^{k}}$, hence $\left|\widehat{h}^{(k)}(b)\right|<\frac{\epsilon}{s^{k}}$. This shows that $\liminf _{\mathcal{V}}\left|h^{(k)}(x)\right|=0$ and therefore $\lim _{\mathcal{V}}\left|h^{(k)}(x)\right|=0$, hence $h^{(k)}$ belongs to $\mathcal{J}(\mathcal{V}) \forall k \in \mathbb{N}$. But since $\mathcal{J}(\mathcal{U})=\mathcal{J}(\mathcal{V}),(\widehat{h})^{(k)}$ belongs to $\mathcal{J}(\mathcal{U})$ for all $k \in \mathbb{N}^{*}$, a contradiction that finishes the proof.

\section{References}

[1] [1] Araujo, J. Prime and maximal ideals in the spectrum of the ultrametric algebra $H^{\infty}(D)$. paper in preparation

[2] [2] Berkovich, V. Spectral Theory and Analytic Geometry over Non-Archimedean Fields. AMS, Providence, (1990)

[3] [3] Boussaf, K. Identity theorem for bounded meromorphic P-adic functions. Bulletin des Sciences Mathématiques.

[4] [4] Carleson, L. Interpolation by bounded analytic functions and the corona problem Annals of Math. 76, p. 547-559 (1962).

[5] [5] Escassut, A. Analytic Elements in p-adic Analysis, World Scientific Publishing Inc., Singapore (1995).

[6] [6] Escassut, A. Ultrametric Banach Algebras, World Scientific Publishing Inc., Singapore (2003).

[7] [7] Escassut, A. and Mainetti, $\mathbf{N}$ About the ultrametric Corona problem Bulletin des Sciences Mathématiques 132, p. 382-394 (2008) 
[8] [8] Escassut, A. and Mainetti, N. On Ideals of the Algebra of p-adic Bounded Analytic Functions on a Disk Bull. Belg. Math. Soc. Simon Stevin 14, 871876 (2007).

[9] [9] Escassut, A Ultrametric Corona Problem and Spherically complete fields Proceedings of the Edinburgh Mathematical Society (Series 2), Volume 53, Issue 02, June 2010, pp 353-371 (2010).

[10] [10] Escassut, A. and Mainetti, N. The ultrametric corona problem. Contemporary Mathematics 508, p.35-45, (2010)

[11] [11] Escassut, A. and Mainetti, N. Multiplicative spectrum of Banach algebras of continuous fonctions. To appear in Topology and its applications.

[12] [12] Guennebaud, B. Sur une notion de spectre pour les algèbres normées ultramétriques, thèse Université de Poitiers, (1973).

[13] [18]Lazard, M. Les zéros des fonctions analytiques sur un corps valué complet, IHES, Publications Mathématiques n14, p.47-75 ( 1962).

[14] [14] Van Der Put, M. The Non-Archimedean Corona Problem Table Ronde Anal. non Archimedienne, Bull. Soc. Math. Mémoire 39-40, p. 287-317 (1974).

Current address: Laboratoire de Mathématiques, UMR 6620, Université Blaise Pascal \& CNRS, Complexe scientifique des Cézeaux, BP 80026, 63171 Aubière Cedex, France

E-mail address: alain.escassut@math.univ-bpclermont.fr 